\title{
Solutions of the Quasi-Vector Wave Equation for Optical Waveguides in a Mapped Infinite Domains by the Galerkin's Method
}

\author{
Kai Ming Lo and E. Herbert Li, Senior Member, IEEE
}

\begin{abstract}
Galerkin's method is employed to analyze the quasivector wave equation for optical waveguides with arbitrary refractive index profile in a mapped infinite domains. Results are presented for a range of waveguide structures which include rectangular core, circular core, rib, and multiple quantum well. Solutions are compared favorably to exact vector solution and numerical results using Fourier operator transform method and beam-propagation method.
\end{abstract}

Index Terms - Eigenvalues/eigenfunctions, Maxwell equations, moment equations, optical propagation, optical waveguides, optical waveguide theory.

\section{INTRODUCTION}

$\mathbf{T}$ HE use of Galerkin's method in solving the scalar wave equation of optical waveguides with arbitrary refractive index profiles was first proposed by Henry and Verbeek [1]. Same method was used by Marcuse in solving the vector wave equation [2]. However, there is a large increase in computing time and memory. In considering that matter, we decide to use the quasi-vector results which include the polarization effects of optical waveguides as an intermediate solution. The memory requirement is the same as in solving the scalar wave equation while the computing time is moderate.

We also employ a mapping scheme to eliminate the need of enclosing waveguide structures within a rectangle whose size affect the accuracy of calculations [3]. Alternately, the elimination can be done by using Hermite-Gauss functions as basis functions [4] rather than sine functions which are used in present studies. However, such elimination is only valid for waveguides with homogeneous cladding. For inhomogeneous cladding waveguides like rib waveguides, the cladding have to be truncated if Hermite-Gauss basis functions are used. On the other hand, no truncation of cladding is needed if sine basis functions are used in a mapped infinite domains.

In the next section, we will derive the quasi-vector wave equation and establish the details of solving this equation using the Galerkin's method. In Section III, we compare some of our numerical results with those of other authors. The

Manuscript received June 18, 1997. This work was supported by the Research Grant Council of Hong Kong. The work of K. M. Lo was supported by the University of Hong Kong Postdoctoral Fellowship.

The authors are with the Department of Electrical and Electronic Engineering, The University of Hong Kong, Hong Kong.

Publisher Item Identifier S 0733-8724(98)03324-6. summary section anticipates the range of potential applications of present method.

\section{MATHEMATICAL FORMULATION}

\section{A. Quasi-Vector Wave Equation}

Maxwell's equations are employed to calculate the spatial variation of electric field $\mathbf{E}(x, y, z)$ and magnetic field $\mathbf{H}(x, y, z)$ of an optical waveguide. The dielectric constant $\varepsilon(x, y, z)$ of a waveguide is related to its refractive index $n(x, y, z)$ by $\varepsilon=n^{2} \varepsilon_{0}$, where $\varepsilon_{0}$ is the free space electric permittivity. The magnetic permeability is taken to have its free space value $\left(\mu=\mu_{0}\right)$ everywhere. The field vectors are taken to depend on time through the implicit factor $\exp (-i \omega t)$. Under these conditions and regions are free of charges and current, Maxwell's equations are written as

$$
\begin{aligned}
& \nabla \times \mathbf{E}=i\left(\mu_{0} / \varepsilon_{0}\right)^{1 / 2} k \mathbf{H} \\
& \nabla \times \mathbf{H}=-i\left(\varepsilon_{0} / \mu_{0}\right)^{1 / 2} k n^{2} \mathbf{E}
\end{aligned}
$$

where $k=2 \pi / \lambda$ is the free space wavenumber, and $\lambda$ is the wavelength of light in free space.

If we eliminate the magnetic field from (1) and (2) by $\nabla \times(1)$ and substitute $\nabla \times \mathbf{H}$ from (2) into, we obtain the vector wave equation

$$
\left(\nabla^{2}+k^{2} n^{2}\right) \mathbf{E}=-\nabla\left(\mathbf{E} \cdot \nabla \ln n^{2}\right)
$$

by using two vector identities, viz.

$$
\begin{aligned}
\nabla \times(\nabla \times \mathbf{E}) & =\nabla(\nabla \cdot \mathbf{E})-\nabla^{2} \mathbf{E} \\
\nabla \cdot\left(n^{2} \mathbf{E}\right) & =n^{2} \nabla \cdot \mathbf{E}+\mathbf{E} \cdot \nabla n^{2}=0
\end{aligned}
$$

The $\nabla^{2}$ in (3) is a vector operator. However, if the field vectors have components referred to fixed Cartesian directions $x, y$, and $z$ as indicated in Fig. 1(a), the vector operator $\nabla^{2}$ is replaced by the scalar Laplacian $\nabla^{2}$. Moreover, if an optical waveguide with refractive index profile that does not change with distance $z$ along the waveguide, i.e., $n=n(x, y)$. The electric field of the waveguide can be written in separable form as

$$
\mathbf{E}(x, y, z)=\mathbf{e}(x, y) \exp (i \beta z)
$$




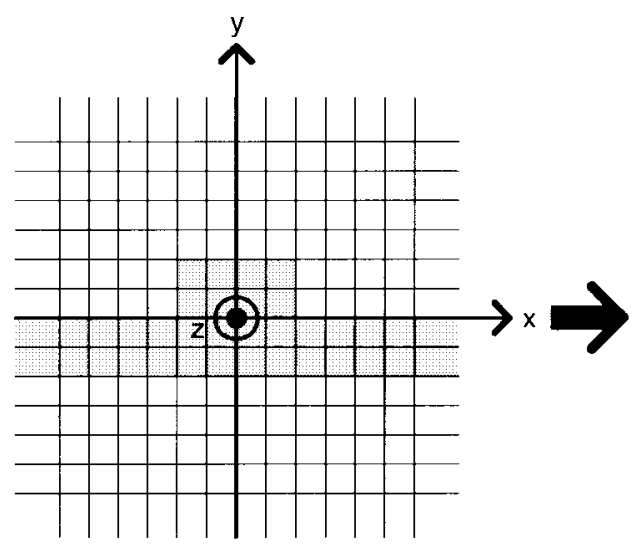

(a)

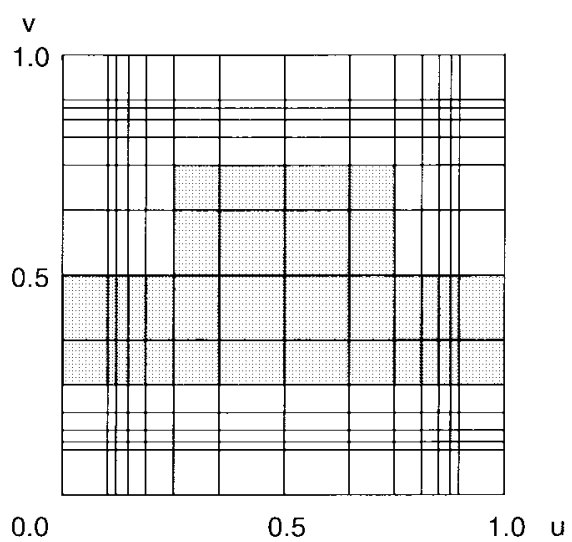

(b)

Fig. 1. An optical structure in (a) $x-y$ plane and (b) transformed $u-v$ domains. A rib waveguide is used as an example.

where $\beta$ is the propagation constant. Thus if we set

$$
\mathbf{e}=e_{x} \hat{\mathbf{x}}+e_{y} \hat{\mathbf{y}}+e_{z} \hat{\mathbf{z}}
$$

in (6) where $\hat{\mathbf{x}}, \hat{\mathbf{y}}$, and $\hat{\mathbf{z}}$ are unit vector parallel to the axes in Fig. 1(a) and using

$$
\nabla^{2}=\nabla^{2}=\frac{\partial^{2}}{\partial x^{2}}+\frac{\partial^{2}}{\partial y^{2}}-\beta^{2}
$$

(3) is reduced to two equations coupling the field components $e_{x}$ and $e_{y}$ as follows:

$$
\begin{gathered}
\frac{\partial^{2} e_{x}}{\partial x^{2}}+\frac{\partial^{2} e_{x}}{\partial y^{2}}+\left(k^{2} n^{2}-\beta^{2}\right) e_{x} \\
+2 \frac{\partial}{\partial x}\left(e_{x} \frac{\partial \ln n}{\partial x}+e_{y} \frac{\partial \ln n}{\partial y}\right)=0 \\
\frac{\partial^{2} e_{y}}{\partial x^{2}}+\frac{\partial^{2} e_{y}}{\partial y^{2}}+\left(k^{2} n^{2}-\beta^{2}\right) e_{y} \\
+2 \frac{\partial}{\partial y}\left(e_{x} \frac{\partial \ln n}{\partial x}+e_{y} \frac{\partial \ln n}{\partial y}\right)=0 .
\end{gathered}
$$

If the coupling terms in (9) and (10) are neglected, we have

$$
\begin{aligned}
& \frac{\partial^{2} e_{x}}{\partial x^{2}}+\frac{\partial^{2} e_{x}}{\partial y^{2}}+\left(k^{2} n^{2}-\beta^{2}\right) e_{x}+2 \frac{\partial}{\partial x}\left(e_{x} \frac{\partial \ln n}{\partial x}\right)=0 \\
& \frac{\partial^{2} e_{y}}{\partial x^{2}}+\frac{\partial^{2} e_{y}}{\partial y^{2}}+\left(k^{2} n^{2}-\beta^{2}\right) e_{y}+2 \frac{\partial}{\partial y}\left(e_{y} \frac{\partial \ln n}{\partial y}\right)=0 .
\end{aligned}
$$

These are in fact the scalar wave equation with polarization correction which are referred here as the quasi-TE wave equation and the quasi-TM wave equation.

The assumption used in (11) and (12), i.e., $e_{x} \gg e_{y}$ in (11) and $e_{y} \gg e_{x}$ in (12), is accurate for three classes of waveguides [5]: 1) weakly guiding waveguides [6] with arbitrary shape and small difference in refractive index between core and cladding or substrate, 2) rectangular core waveguides with arbitrary core-cladding refractive index operated in the far-from cutoff region, and 3) arbitrary refractive index profile waveguides with an elongated or slab like cross section. Numerical results for these three classes of waveguides will be given in Section III.

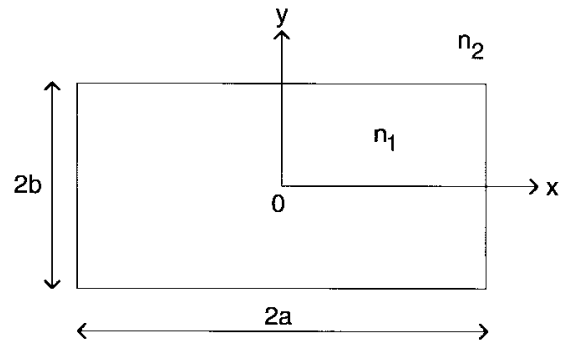

Fig. 2. Structure of a rectangular core optical waveguide.

\section{B. Galerkin's Method}

Since the formulation of the quasi-vector wave (11) and (12) are the same, the quasi-TE wave (11) will be solved in below using Galerkin's method and the procedure developed is applicable for solving the quasi-TM wave equation (12).

To eliminate the need of enclosing waveguide within a rectangle, the whole $x-y$ plane is mapped onto a unit square in $u-v$ space as shown in Fig. 1 using the transformation functions

$$
\begin{aligned}
& x=\alpha_{x} \tan \left[\pi\left(u-\frac{1}{2}\right)\right] \\
& y=\alpha_{y} \tan \left[\pi\left(v-\frac{1}{2}\right)\right]
\end{aligned}
$$

where $\alpha_{x}$ and $\alpha_{y}$ are scaling parameters in the $x$ and $y$ directions, respectively. The same change of variables is applied to the quasi-TE wave equation (11) and in the $u-v$ space it is written as

$$
\begin{aligned}
& \left(\frac{d u}{d x}\right)^{2} \frac{\partial^{2} e_{x}}{\partial u^{2}}+\frac{d^{2} u}{d x^{2}} \frac{\partial e_{x}}{\partial u}+\left(\frac{d v}{d y}\right)^{2} \frac{\partial^{2} e_{x}}{\partial v^{2}}+\frac{d^{2} v}{d y^{2}} \frac{\partial e_{x}}{\partial v} \\
& +\left(n^{2} k^{2}-\beta^{2}\right) e_{x}+2\left(\frac{d u}{d x}\right)^{2} \frac{\partial}{\partial u}\left(e_{x} \frac{\partial \ln n}{\partial u}\right) \\
& +2 \frac{d^{2} u}{d x^{2}} e_{x} \frac{\partial \ln n}{\partial u}=0
\end{aligned}
$$

where $e_{x}=e_{x}(u, v), n=n(u, v)$. 


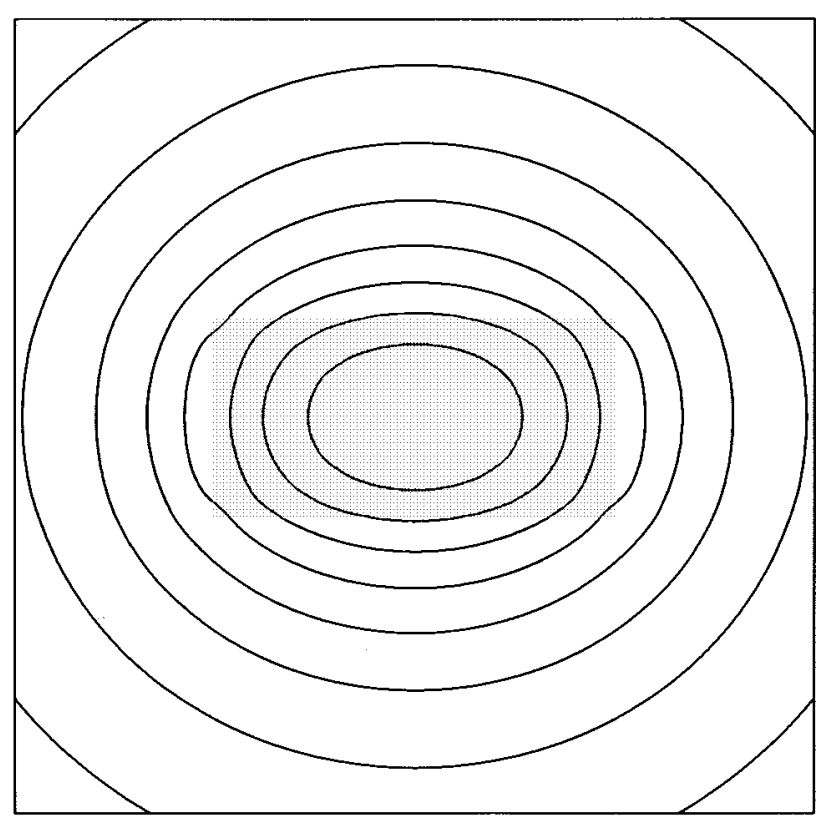

(a)

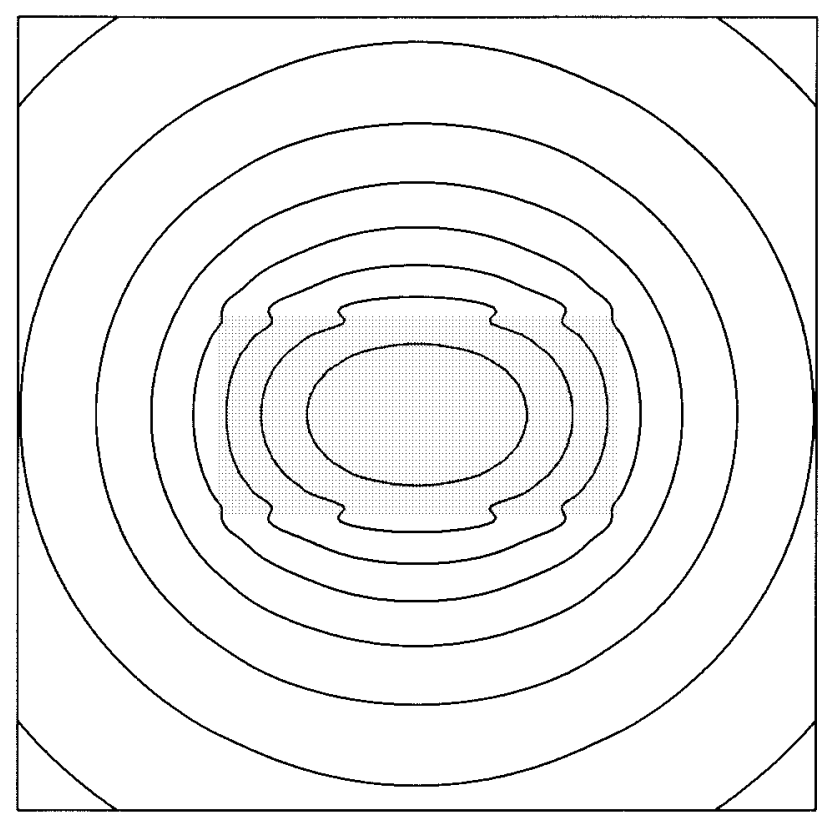

(b)

Fig. 3. Field pattern of (a) $e_{x}$ of $\mathrm{E}_{11}^{\mathrm{x}}$ mode and (b) $e_{y}$ of $\mathrm{E}_{11}^{\mathrm{y}}$ mode at $2 V_{b} / \pi=0.5$ for $N=25$. The core region is shaded. Contour lines are at $10 \%$ intervals of peak amplitude.

The unknown electric field component $e_{x}$ is expanded as

$$
e_{x}=\sum_{i}^{N_{m} N_{n}} a_{i} \phi_{i}(u, v)=\sum_{m_{i}=1}^{N_{m}} \sum_{n_{i}=1}^{N_{n}} a_{m_{i}, n_{i}} \phi_{i}(u, v)
$$

where integer quotient function div and remainder on division function mod are employed to relate the index, $i$, and spatial frequencies, $m_{i}$ and $n_{i}$, as

$$
\begin{aligned}
m_{i} & =(i-1) \operatorname{div} N_{n}+1 \\
n_{i} & =(i-1) \bmod N_{n}+1 .
\end{aligned}
$$

TABLE I

$P^{2}$ AS a Function of $2 V_{b} / \pi$ For the Fundamental Quasi-Vector Modes OF the Rectangular Core Optical Waveguide. $\alpha_{x}=a, \alpha_{y}=b, \rho=\sqrt{a b}$,

\begin{tabular}{|c|c|c|c|c|c|}
\hline \multirow[t]{2}{*}{$2 V_{b} / \pi$} & \multicolumn{4}{|c|}{$N$} & \multirow[t]{2}{*}{ F-OPT } \\
\hline & 10 & 15 & 20 & 25 & \\
\hline \multicolumn{6}{|c|}{$\mathrm{E}_{11}^{\mathrm{x}}$ mode } \\
\hline 0.40 & 0.0346 & 0.0343 & 0.0337 & 0.0335 & 0.0400 \\
\hline 0.45 & 0.0679 & 0.0664 & 0.0661 & 0.0661 & 0.0670 \\
\hline 0.50 & 0.1077 & 0.1069 & 0.1069 & 0.1069 & 0.1068 \\
\hline 0.55 & 0.1523 & 0.1522 & 0.1523 & 0.1522 & 0.1520 \\
\hline 0.60 & 0.1991 & 0.1993 & 0.1992 & 0.1992 & 0.1990 \\
\hline 0.65 & 0.2458 & 0.2459 & 0.2459 & 0.2458 & 0.2456 \\
\hline 0.70 & 0.2909 & 0.2910 & 0.2910 & 0.2909 & 0.2907 \\
\hline 0.75 & 0.3338 & 0.3339 & 0.3338 & 0.3338 & 0.3336 \\
\hline 0.80 & 0.3741 & 0.3742 & 0.3741 & 0.3741 & 0.3739 \\
\hline 0.85 & 0.4117 & 0.4118 & 0.4117 & 0.4117 & 0.4116 \\
\hline 0.90 & 0.4467 & 0.4467 & 0.4467 & 0.4467 & 0.4465 \\
\hline 0.95 & 0.4791 & 0.4791 & 0.4791 & 0.4791 & 0.4789 \\
\hline 1.00 & 0.5090 & 0.5091 & 0.5091 & 0.5091 & 0.5089 \\
\hline \multicolumn{6}{|c|}{$E_{11}^{y}$ mode } \\
\hline 0.40 & 0.0274 & 0.0315 & 0.0320 & 0.0307 & 0.0483 \\
\hline 0.45 & 0.0603 & 0.0617 & 0.0614 & 0.0614 & 0.0612 \\
\hline 0.50 & 0.0999 & 0.1004 & 0.1000 & 0.1005 & 0.1003 \\
\hline 0.55 & 0.1444 & 0.1443 & 0.1440 & 0.1444 & 0.1441 \\
\hline 0.60 & 0.1908 & 0.1903 & 0.1901 & 0.1902 & 0.1900 \\
\hline 0.65 & 0.2370 & 0.2363 & 0.2361 & 0.2361 & 0.2359 \\
\hline 0.70 & 0.2817 & 0.2810 & 0.2809 & 0.2808 & 0.2805 \\
\hline 0.75 & 0.3243 & 0.3237 & 0.3236 & 0.3235 & 0.3232 \\
\hline 0.80 & 0.3645 & 0.3640 & 0.3639 & 0.3638 & 0.3635 \\
\hline 0.85 & 0.4021 & 0.4018 & 0.4017 & 0.4016 & 0.4013 \\
\hline 0.90 & 0.4372 & 0.4370 & 0.4369 & 0.4368 & 0.4365 \\
\hline 0.95 & 0.4698 & 0.4697 & 0.4696 & 0.4695 & 0.4692 \\
\hline 1.00 & 0.5001 & 0.5000 & 0.5000 & 0.4998 & 0.4996 \\
\hline
\end{tabular}
AND $N_{m}^{e}=N_{n}^{e}=N$. The PARAmeters $N_{m}^{e}$ AND $N_{n}^{e}$ IndicAte the Number of Even Spatial Frequency Components Used in the $x$ AND $y$ Directions

The expansion functions $\phi_{i}(u, v)$ are chosen as the complete set of orthonormal sine basis functions as

$$
\phi_{i}(u, v)=2 \sin \left(m_{i} \pi u\right) \sin \left(n_{i} \pi v\right) .
$$

The field expansion (16) is substituted into (15), it is then multiplied by $\phi_{j}(u, v)$ and integrated over the unit square in Fig. 1(b) and yield the result

$$
\sum_{i}^{N_{m} N_{n}}\left(S_{j, i}+P_{j, i}-W^{2} \delta_{j, i}\right) a_{i}=0
$$

where

$$
S_{j, i}=V^{2} A_{j, i}+B_{j, i}
$$

correspond to the scalar wave equation with

$$
\begin{aligned}
A_{j, i} & =\int_{u=0}^{1} \int_{v=0}^{1} g(u, v) \phi_{i}(u, v) \phi_{j}(u, v) d u d v \\
B_{j, i} & =\rho^{2}\left(I_{1}+I_{2}+I_{3}+I_{4}\right)
\end{aligned}
$$




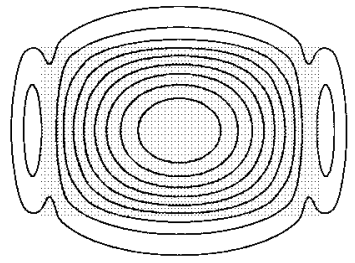

(a)

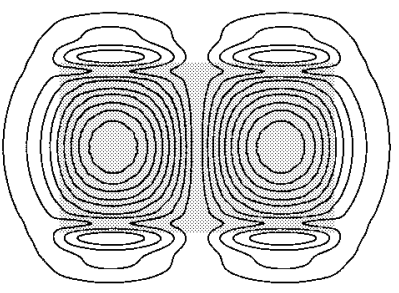

(c)

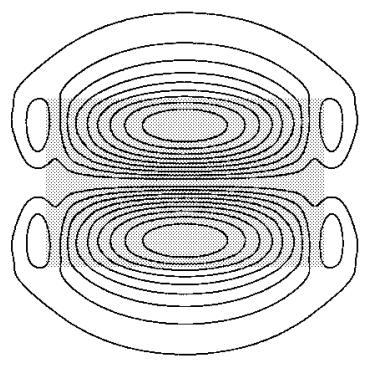

(e)

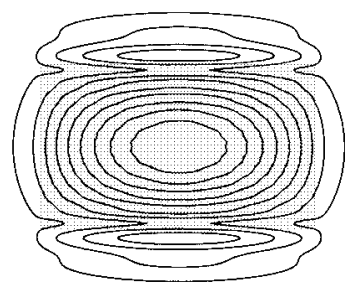

(b)

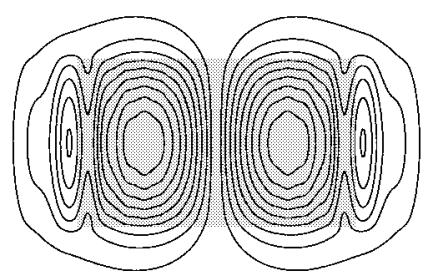

(d)

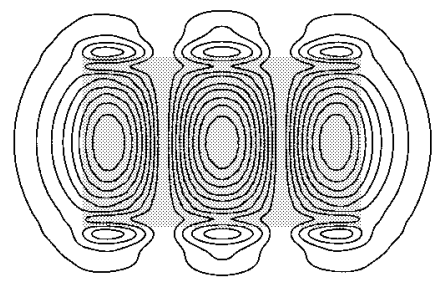

(f)
Fig. 4. (a)-(f) Field patterns, $e_{x}$ for quasi-TE mode and $e_{y}$ for quasi-TM mode, of the first six bound modes of the high contrast rectangular core waveguide for $N=30$. Designation of the modes are given in Table II.

TABLE II

$P^{2}$ of the First Six Bound Modes of the High Refractive Index Contrast Rectangular Core Waveguide. $N_{m}^{e}=N_{n}^{e}=N$ for Modes (a), (b), AND (f); $N_{m}^{o}=N_{n}^{e}=N$ FOR MODES (c) AND (d); AND $N_{m}^{e}=N_{n}^{o}=N$ for Mode (e). The Parameters $N_{m}^{e, o}$ AND $N_{n}^{e}{ }^{o}$ Indicate the Number of EVEn AND OdD SPatial Frequency COMPONENTS UsED IN THE $x$ AND $y$ DiReCTIONS, ResPeCTIVELY

\begin{tabular}{ccccccc}
\hline \multirow{2}{*}{$N$} & \multicolumn{6}{c}{ Mode } \\
\cline { 2 - 7 } & (a) & (b) & (c) & (d) & (c) & (f) \\
& $\mathrm{E}_{11}^{\mathrm{x}}$ & $\mathrm{E}_{11}^{y}$ & $\mathrm{E}_{21}^{\mathrm{y}}$ & $\mathrm{E}_{21}^{\mathrm{x}}$ & $\mathrm{E}_{12}^{\mathrm{x}}$ & $\mathrm{E}_{31}^{\mathrm{y}}$ \\
\hline 10 & 0.7591 & 0.7007 & 0.5041 & 0.5000 & 0.3390 & 0.1979 \\
15 & 0.7585 & 0.6968 & 0.5008 & 0.4991 & 0.3386 & 0.1961 \\
20 & 0.7584 & 0.6972 & 0.5012 & 0.4961 & 0.3386 & 0.1965 \\
25 & 0.7580 & 0.6953 & 0.4995 & 0.4962 & 0.3382 & 0.1950 \\
30 & 0.7580 & 0.6960 & 0.5001 & 0.4947 & 0.3383 & 0.1955 \\
$\mathrm{H}-\mathrm{G}[4]$ & 0.7577 & 0.6949 & 0.4988 & 0.4985 & 0.3375 & 0.1942 \\
\hline
\end{tabular}

and

$$
g(u, v)=\frac{n^{2}(u, v)-n_{\mathrm{cl}}^{2}}{n_{\mathrm{co}}^{2}-n_{\mathrm{cl}}^{2}} .
$$

Here, $\delta_{j, i}$ is a delta function and defined as

$$
\delta_{j, i}= \begin{cases}1 & \text { if } j=i \\ 0 & \text { if } j \neq i\end{cases}
$$

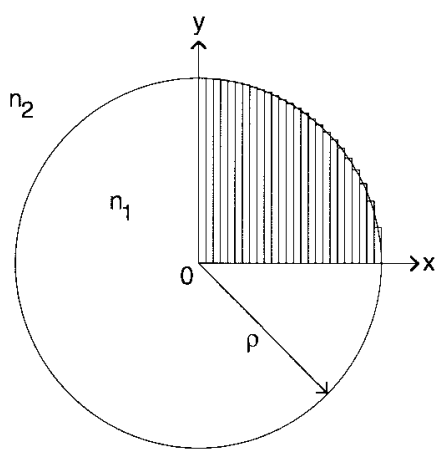

Fig. 5. Structure of a circular core optical fiber.

The modal parameters $V$ and $W$ are defined as

$$
\begin{gathered}
V=k \rho\left(n_{\mathrm{co}}^{2}-n_{\mathrm{cl}}^{2}\right)^{1 / 2} \\
W=\rho\left(\beta^{2}-k^{2} n_{\mathrm{cl}}^{2}\right)^{1 / 2} .
\end{gathered}
$$

The core and cladding refractive index, $n_{\mathrm{co}}$ and $n_{\mathrm{cl}}$, and normalization parameter $\rho$ are chosen to be representative of the refractive index profile of the optical waveguide under consideration. Moreover

$$
P_{j, i}=\rho^{2}\left(I_{5}+I_{6}\right)
$$

correspond to polarization correction. The six integrals $I_{1}$ to $I_{6}$ is given in the Appendix as (29)-(34) and can be evaluated analytically in terms of sum of trigonometric functions if the refractive index profile $n(x, y)$ is approximated by rectangles of uniform refractive index.

The double summation series in (20) can be written as a matrix eigenvalue equation, $\mathbf{M a}=W^{2} \mathbf{a}$, by defining a vector a consisting of the elements $a_{i}$ and a matrix $\mathbf{M}$ composed of the coefficients $S_{j, i}$ and $P_{j, i}$. LAPACK subroutines are used to solve this equation [7], the propagation constants of the bound modes of a waveguide are calculated from the real, positive eigenvalues $W^{2}$ and the corresponding modal field is calculated via the Fourier coefficients of associated eigenvectors a.

\section{NUMERICAL RESUltS}

\section{A. Rectangular Core Optical Waveguide}

Fig. 2 shows an optical waveguide with a rectangular core of width $\mathbf{2 a}$ and height $\mathbf{2 b}$. The core and cladding refractive index are $n_{\mathrm{co}}=n_{1}=1.5$ and $n_{\mathrm{cl}}=n_{2}=1.45 . \lambda=1.15 \mu \mathrm{m}$ and $a / b=2$. Table I gives the normalized propagation constants $P^{2}=\left[(\beta / k)^{2}-n_{\mathrm{cl}}^{2}\right] /\left(n_{\mathrm{co}}^{2}-n_{\mathrm{cl}}^{2}\right)$ for the fundamental quasivector modes as a function of $2 V_{b} / \pi$ where $V_{b}=k b\left(n_{1}^{2}-\right.$ $\left.n_{2}^{2}\right)^{1 / 2}$. The bound modes are denoted as $\mathrm{E}_{\mathrm{mn}}^{\mathrm{x}}$ mode for the quasi-TE mode and $\mathrm{E}_{\mathrm{mn}}^{\mathrm{y}}$ mode for the quasi-TM mode, respectively. The $m$ and $n$ are both positive integers meaning $m-1$ and $n-1$ field zeros in the $x$ and $y$ directions of the modal fields, respectively. The calculated results are in excellent agreement with Fourier operator transform (F-OPT) method [8] except at $2 V_{b} / \pi=0.4$ where present method is more accurate as shown by the convergence of $P^{2}$. The modal fields at $2 V_{b} / \pi=0.5$ is shown in Fig. 3. As can be clearly seen 
TABLE III

$P^{2}$ AS a Function of Fiber Parameter $V=k \rho\left(n_{1}^{2}-n_{2}^{2}\right)^{1 / 2}$ For the Fundamental Quasi-Vector Modes of the Circular CORE OpticAl FibER. $\alpha_{x}=\alpha_{y}=\rho$ AND $N_{m}^{e}=N_{n}^{e}=N$

\begin{tabular}{|c|c|c|c|c|c|c|}
\hline \multirow[t]{4}{*}{$V$} & \multicolumn{6}{|c|}{ Mode } \\
\hline & \multicolumn{4}{|c|}{$\mathrm{E}_{11}^{\mathrm{x}}$} & \multirow{3}{*}{$\begin{array}{c}E_{11}^{\mathrm{y}} \\
N \\
=25\end{array}$} & \multirow[t]{3}{*}{$\mathrm{HE}_{11}$} \\
\hline & \multicolumn{4}{|c|}{$N$} & & \\
\hline & 10 & 15 & 20 & 25 & & \\
\hline 0.8 & - & 0.0043 & 0.0049 & 0.0046 & 0.0047 & 0.0043 \\
\hline 1.0 & 0.0339 & 0.0330 & 0.0325 & 0.0325 & 0.0325 & 0.0322 \\
\hline 1.2 & 0.0920 & 0.0918 & 0.0918 & 0.0918 & 0.0918 & 0.0911 \\
\hline 1.4 & 0.1696 & 0.1692 & 0.1691 & 0.1690 & 0.1689 & 0.1681 \\
\hline 1.6 & 0.2510 & 0.2506 & 0.2505 & 0.2504 & 0.2503 & 0.2494 \\
\hline 1.8 & 0.3286 & 0.3283 & 0.3281 & 0.3280 & 0.3280 & 0.3270 \\
\hline 2.0 & 0.3991 & 0.3988 & 0.3987 & 0.3986 & 0.3985 & 0.3976 \\
\hline 2.2 & 0.4615 & 0.4613 & 0.4612 & 0.4611 & 0.4610 & 0.4603 \\
\hline 2.4 & 0.5163 & 0.5161 & 0.5160 & 0.5159 & 0.5158 & 0.5151 \\
\hline 2.6 & 0.5640 & 0.5639 & 0.5638 & 0.5637 & 0.5636 & 0.5630 \\
\hline 2.8 & 0.6056 & 0.6055 & 0.6054 & 0.6054 & 0.6053 & 0.6048 \\
\hline 3.0 & 0.6420 & 0.6419 & 0.6418 & 0.6418 & 0.6417 & 0.6412 \\
\hline
\end{tabular}

the present method is able to reproduce the discontinuity in the field at the core-cladding boundaries (vertical side walls for quasi-TE mode and horizontal side walls for quasi-TM mode).

Results of $P^{2}$ for the first six bound modes of another rectangular core waveguide with high refractive index contrast $\left(n_{1}=2, n_{2}=1\right)$ is shown in Table II. The associated field of these modes are shown in Fig. 4. Here, $V_{b}=3$ and $a / b=5 / 3$. Results of $P^{2}$ are in good agreement with results using Hermite-Gauss (H-G) basis functions [4]. As is expected, this present method, with sine basis functions in the transformed domains, has field zero at infinity as does Hermite-Gauss basis functions.

\section{B. Step-Index Core Optical Fiber}

The next structure to be studied is a circular core optical fiber (Fig. 5) with $n_{\mathrm{co}}=n_{1}=1.6$ and $n_{\mathrm{cl}}=n_{2}=$ 1.5. The first quadrant of the circular core is resembled by 25 uniform rectangles and the rest is found by symmetry. $P^{2}$ of the two fundamental quasi-vector modes are almost the same as listed in Table III. Theoretically, with infinite number of rectangles, the fundamental quasi-vector modes are degenerated. Results of present quasi-vector solutions are compared with the exact vector solutions [6], $\mathrm{HE}_{11}$ mode, and they are in good agreement.

The field patterns of the quasi-vector modes at $V=1$ is given in Fig. 6. Discontinuity of field is clearly seen at the core-cladding boundary. The field component $e_{x}$ of $\mathrm{E}_{11}^{\mathrm{x}}$ mode along the $x$ axis as a function of normalized distance $X=x / \rho$ is shown in Fig. 7(a). As can be seen from the figure, the quasivector modal fields are agree with the exact vector solution except near the core-cladding boundary which is detailed in Fig. 7(b). From this figure, $e_{x}$ of quasi-TE mode near the

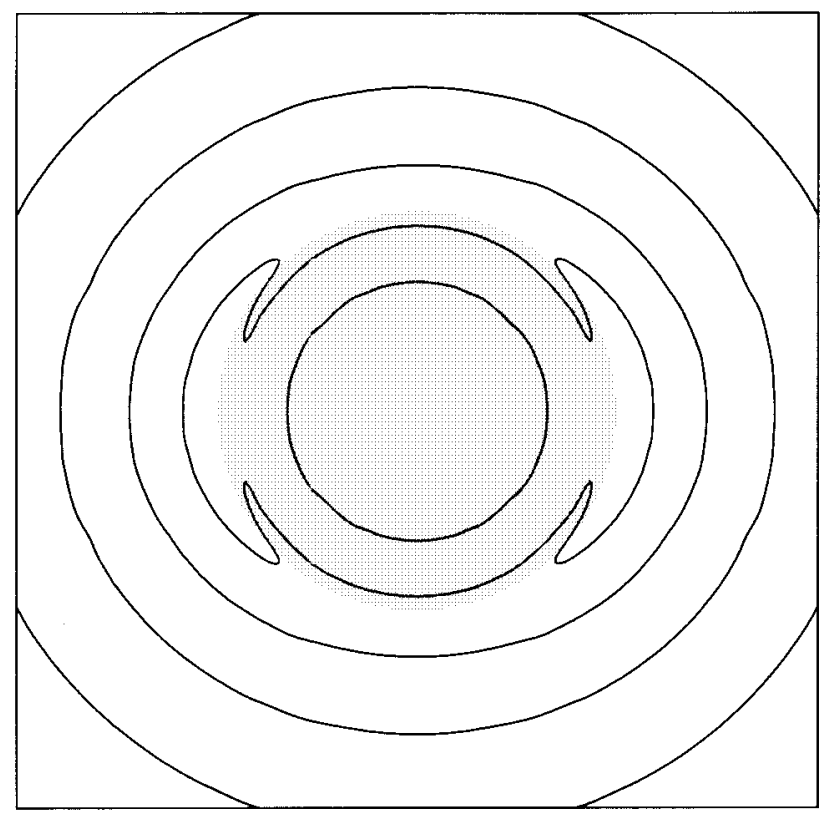

(a)

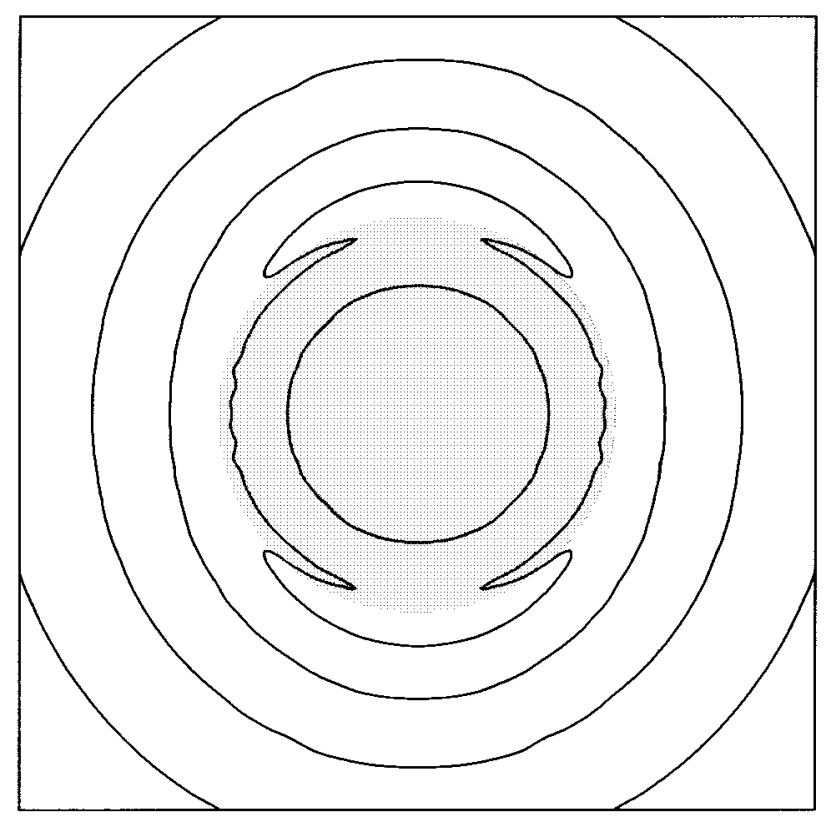

(b)

Fig. 6. Field patterns of (a) $e_{x}$ of $\mathrm{E}_{11}^{\mathrm{x}}$ mode and (b) $e_{y}$ of $\mathrm{E}_{11}^{\mathrm{y}}$ mode of the circular core optical fiber at $V=1$ for $N=25$.

boundary is likely to be an average of the exact vector solution and it is converge moderately to the exact vector solution as $N$ is increased.

\section{Rib Optical Waveguide}

A rib optical waveguide as shown in Fig. 8 is considered in this section. Here, $n_{\mathrm{co}}=n_{1}=3.44, n_{\mathrm{cl}}=n_{2}=3.4, n_{3}=1$, $t=1 \mu \mathrm{m}, w=3 \mu \mathrm{m}$, and $\lambda=1.15 \mu \mathrm{m}$. Table IV shows $P^{2}$ for the fundamental quasi-vector modes as a function of $d$. By comparison with results from F-OPT method [8] and beam propagation method (BPM) [9], the convergence of present solutions is better for $E_{11}^{x}$ mode than $E_{11}^{y}$ mode. This could be 


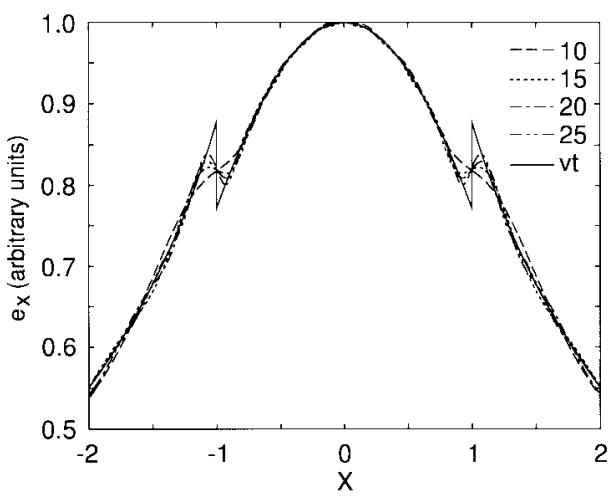

(a)

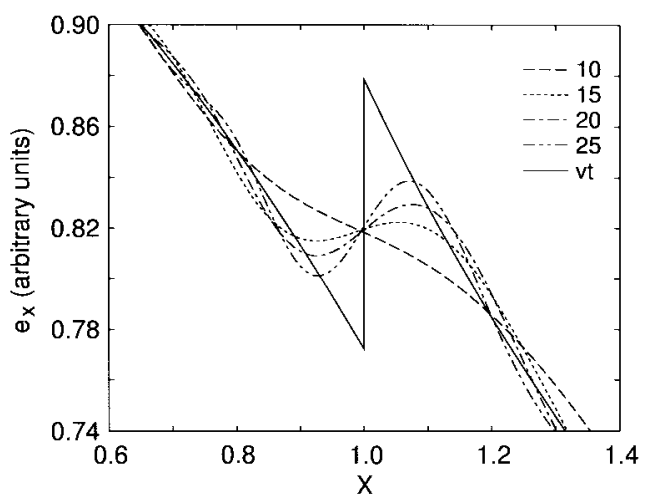

(b)

Fig. 7. (a) Electric field component $e_{x}$ of quasi-TE mode as a function of normalized distance $X$ for $N=10,15,20$, and 25 . The electric field component $e_{x}$ of the exact vector (vt) solution is given in solid line. (b) Details of (a) near $X=1$.

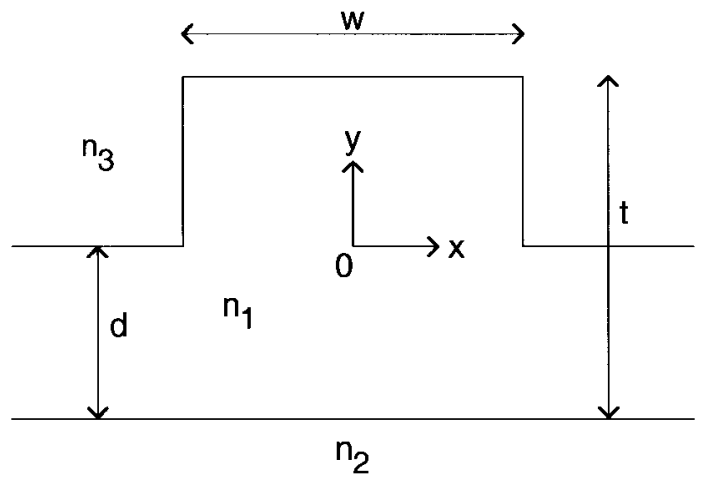

Fig. 8. Structure of a rib optical waveguide.

explained by the fact that higher spatial frequency components are needed to model the large discontinuity of field component $e_{y}$ of $\mathrm{E}_{11}^{\mathrm{y}}$ mode at the core-air interfaces perpendicular to the $y$ axis of Fig. 8 which are clearly seen form the field patterns at Fig. 9.

\section{IID MQW Waveguide}

The last example is to apply present method to a multiple quantum-well (MQW) waveguide fabricated by impurityinduced disordering (IID) technique [10]. The IID technique provides an efficient way to realize waveguiding structure

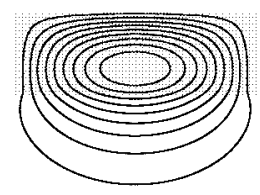

(a)

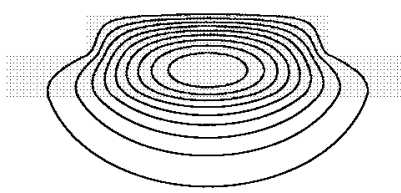

(c)

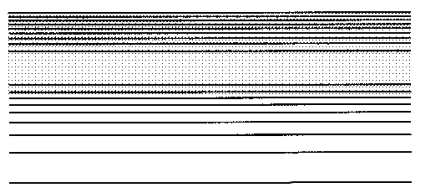

(e)

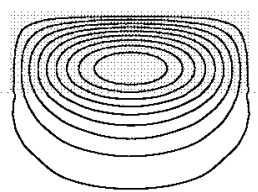

(b)

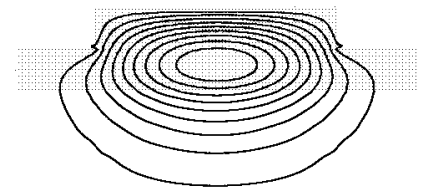

(d)

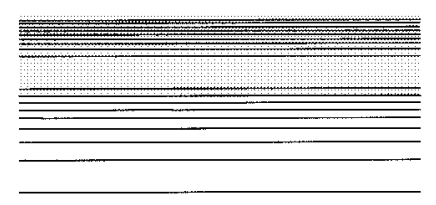

(f)
Fig. 9. Field patterns $e_{x}$ of $\mathrm{E}_{11}^{\mathrm{x}}$ mode (left side) and $e_{y}$ of $\mathrm{E}_{11}^{\mathrm{y}}$ mode (right side), of the rib waveguide for $N=30$ at (a)-(b) $d=0 \mu \mathrm{m}$, (c)-(d) $d=0.5 \mu \mathrm{m}$, and (e)-(f) $d=1 \mu \mathrm{m}$.

in optoelectronic integrated circuits [11]. The structure to be modeled consists of $\mathrm{Al}_{0.3} \mathrm{Ga}_{0.7} \mathrm{As} / \mathrm{GaAs} \mathrm{QW}$ layers and thick $\mathrm{Al}_{0.3} \mathrm{Ga}_{0.7} \mathrm{As}$ buffer layer grown on a GaAs substrate; the schematic of the structure is shown in Fig. 10. In our model, $\mathrm{Ga}^{+}$ion is implanted with a projected range located around the center of the QW layers. The implantation process produces a modification of the $\mathrm{QW}$ material which in turn leads to differences in refractive index in different region [10]. The implanted region has a lower refractive index than the nonimplanted region, hence produce lateral confinement of light. Fig. 11 shows a IID MQW refractive index profile which is represented by rectangles of constant refractive index. The profile was calculated for $2 a=3 \mu \mathrm{m}, 2 b=$ $0.6 \mu \mathrm{m}$ at $\lambda=0.901085 \mu \mathrm{m}$. The maximum and minimum refractive index are assigned as $n_{\mathrm{co}}(=3.52695)$ and $n_{\mathrm{cl}}(=$ 3.46782 ), respectively. Other parameters are refractive index of $\mathrm{Al}_{0.3} \mathrm{Ga}_{0.7} \mathrm{As}$ buffer layer and air cover which is equal to 3.35447 and 1 , respectively. $P^{2}$ of the fundamental quasivector modes are listed in Table $\mathrm{V}$. The associated modal fields are shown in Fig. 12. Results show that present method is applicable for finding the waveguiding properties of an optical waveguide with a diffused refractive index profile.

\section{CONCLUSIONS}

A numerical method for solving the guided quasi-vector modes problem of optical waveguide with arbitrary refractive index in a mapped infinite domains is described. Solving the problem with the Galerkin's method, the mode field is expanded into a two-dimensional (2-D) Fourier sine series and resulting in a matrix eigenvalue equation which is solved using the LAPACK subroutines.

The accuracy of present method is compared to the Fourier operator transform method and the beam propagation method for a rectangular core waveguide and a rib waveguide. Moreover, solutions for step-index circular core optical fiber are 
TABLE IV

$P^{2}$ AS A Function of $d$ FOR Fundamental QuASI-VeCtor Modes of the Rib Optical WaVeguide. $\alpha_{x}=w / 2$, $\alpha_{y}=t / 2, \rho=\sqrt{\alpha_{x} \alpha_{y}}, N_{m}^{e}=N$ AND $N_{n}=2 N$

\begin{tabular}{|c|c|c|c|c|c|}
\hline \multirow[t]{2}{*}{$d$} & \multicolumn{3}{|c|}{$N$} & \multirow[t]{2}{*}{ F-OPT } & \multirow[t]{2}{*}{$\mathrm{BPM}$} \\
\hline & 10 & 20 & 30 & & \\
\hline \multicolumn{6}{|c|}{$E_{11}^{\mathrm{x}}$ mode } \\
\hline 0.0 & 0.2879 & 0.2961 & 0.2971 & 0.2992 & 0.3001 \\
\hline 0.1 & 0.2911 & 0.2989 & 0.2997 & 0.3018 & 0.3026 \\
\hline 0.2 & 0.2957 & 0.3029 & 0.3036 & 0.3055 & 0.3066 \\
\hline 0.3 & 0.3019 & 0.3084 & 0.3091 & 0.3108 & 0.3116 \\
\hline 0.4 & 0.3101 & 0.3158 & 0.3163 & 0.3178 & 0.3188 \\
\hline 0.5 & 0.3202 & 0.3251 & 0.3255 & 0.3267 & 0.3269 \\
\hline 0.6 & 0.3324 & 0.3364 & 0.3368 & 0.3373 & 0.3380 \\
\hline 0.7 & 0.3468 & 0.3500 & 0.3503 & 0.3509 & 0.3504 \\
\hline 0.8 & 0.3639 & 0.3666 & 0.3667 & 0.3668 & 0.3655 \\
\hline 0.9 & 0.3859 & 0.3880 & 0.3886 & 0.3880 & 0.3871 \\
\hline 1.0 & 0.4241 & 0.4268 & 0.4271 & 0.4273 & 0.4273 \\
\hline \multicolumn{6}{|c|}{$\mathrm{E}_{11}^{\mathrm{y}}$ mode } \\
\hline 0.0 & 0.2495 & 0.2559 & 0.2567 & 0.2652 & 0.2664 \\
\hline 0.1 & 0.2523 & 0.2581 & 0.2588 & 0.2678 & 0.2685 \\
\hline 0.2 & 0.2561 & 0.2614 & 0.2619 & 0.2703 & 0.2720 \\
\hline 0.3 & 0.2615 & 0.2659 & 0.2664 & 0.2746 & 0.2762 \\
\hline 0.4 & 0.2682 & 0.2719 & 0.2723 & 0.2804 & 0.2823 \\
\hline 0.5 & 0.2769 & 0.2798 & 0.2800 & 0.2880 & 0.2892 \\
\hline 0.6 & 0.2877 & 0.2896 & 0.2898 & 0.2976 & 0.2990 \\
\hline 0.7 & 0.3002 & 0.3016 & 0.3017 & 0.3095 & 0.3101 \\
\hline 0.8 & 0.3161 & 0.3166 & 0.3166 & 0.3244 & 0.3237 \\
\hline 0.9 & 0.3367 & 0.3370 & 0.3367 & 0.3446 & 0.3441 \\
\hline 1.0 & 0.3765 & 0.3769 & 0.3769 & 0.3851 & 0.3854 \\
\hline
\end{tabular}

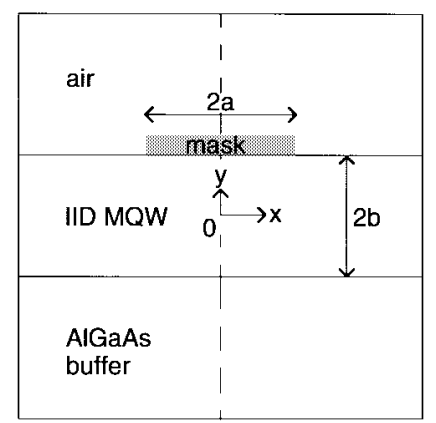

Fig. 10. Schematic of an IID MQW waveguide.

compared with the exact vector solution. Results shown that the present quasi-vector solutions provide a good approximation of the exact solution.

The application of present method to a waveguide with diffused refractive index profile is demonstrated using an MQW waveguide fabricated by impurity induced disordering technique. The normalized propagation constants of the fundamental quasi-vector modes converge moderately as we increase the number of spatial frequencies.

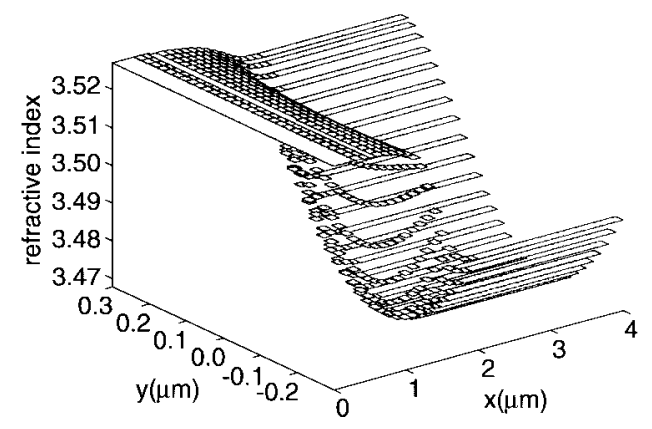

Fig. 11. Refractive index profile (half symmetry) of the IID MQW waveguide.

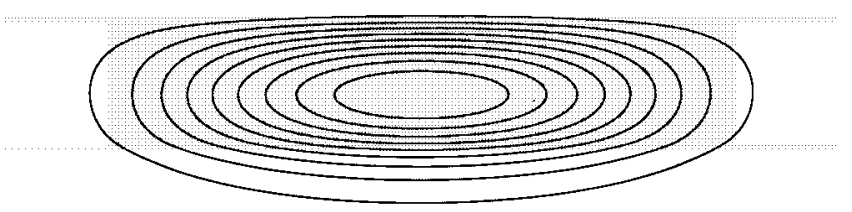

(a)

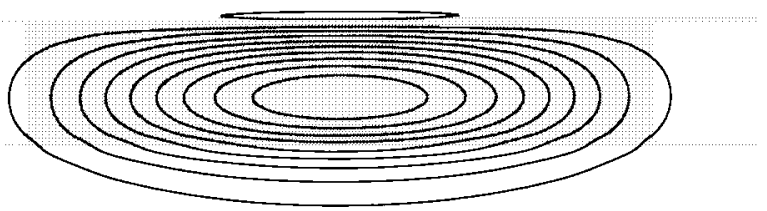

(b)

Fig. 12. Field patterns of (a) $e_{x}$ of $\mathrm{E}_{11}^{\mathrm{x}}$ mode and (b) $e_{y}$ of $\mathrm{E}_{11}^{\mathrm{y}}$ mode of the IID MQW waveguide for $N=25$. The shaded region is the MQW under the mask.

TABLE V

$P^{2}$ FOR the Fundamental Quasi-Vector Modes of the IID MQW WAVEGUIDE. $\alpha_{x}=a, \alpha_{y}=b$, AND $\rho=\sqrt{a b}$

\begin{tabular}{cccc}
\hline$N_{m}^{e}$ & $N_{n}$ & \multicolumn{2}{c}{ Mode } \\
\cline { 3 - 4 } & & $\mathrm{E}_{11}^{\mathrm{x}}$ & $\mathrm{E}_{11}^{y}$ \\
\hline 10 & 20 & 0.1352 & 0.0110 \\
15 & 30 & 0.1375 & 0.0111 \\
20 & 40 & 0.1380 & 0.0096 \\
25 & 50 & 0.1382 & 0.0094 \\
\hline
\end{tabular}

\section{APPENDIX}

The integrals $I_{1}$ to $I_{6}$ in (23) and (28) are given as follows:

$$
\begin{gathered}
I_{1}=-m_{i}^{2} \pi^{2} \int_{u=0}^{1} \int_{v=0}^{1}\left(\frac{d u}{d x}\right)^{2} \phi_{i}(u, v) \phi_{j}(u, v) d u d v \\
=-\frac{m_{i}^{2}}{2 \alpha_{x}^{2}}\left\{\frac{3 \delta_{m_{i}, m_{j}}}{4}-\frac{\delta_{m_{i}, m_{j}-2}}{2}-\frac{\delta_{m_{i}, m_{j}+2}}{2}\right. \\
+\frac{\delta_{m_{i}, 2-m_{j}}}{2}+\frac{\delta_{m_{i}, m_{j}-4}}{8}+\frac{\delta_{m_{i}, m_{j}+4}}{8} \\
\left.\quad-\frac{\delta_{m_{i}, 4-m_{j}}}{8}\right\}_{n_{i}, n_{j}}, \\
I_{2}=m_{i} \pi \int_{u=0}^{1} \int_{v=0}^{1}\left(\frac{d^{2} u}{d x^{2}}\right) \frac{1}{\tan \left(m_{i} \pi u\right)} \\
\quad \times \phi_{i}(u, v) \phi_{j}(u, v) d u d v
\end{gathered}
$$




$$
\begin{aligned}
& =\frac{m_{i}}{\alpha_{x}^{2}}\left\{\frac{\delta_{m_{i}, 2-m_{j}}}{4}+\frac{\delta_{m_{i}, m_{j}-2}}{4}-\frac{\delta_{m_{i}, m_{j}+2}}{4}\right. \\
& \left.-\frac{\delta_{m_{i}, 4-m_{j}}}{8}-\frac{\delta_{m_{i}, m_{j}-4}}{8}+\frac{\delta_{m_{i}, m_{j}+4}}{8}\right\} \delta_{n_{i}, n_{j}} \\
& I_{3}=-n_{i}^{2} \pi^{2} \int_{u=0}^{1} \int_{v=0}^{1}\left(\frac{d v}{d y}\right)^{2} \phi_{i}(u, v) \phi_{j}(u, v) d u d v \\
& =-\frac{n_{i}^{2}}{2 \alpha_{y}^{2}}\left\{\frac{3 \delta_{n_{i}, n_{j}}}{4}-\frac{\delta_{n_{i}, n_{j}-2}}{2}-\frac{\delta_{n_{i}, n_{j}+2}}{2}\right. \\
& +\frac{\delta_{n_{i}, 2-n_{j}}}{2}+\frac{\delta_{n_{i}, n_{j}-4}}{8}+\frac{\delta_{n_{i}, n_{j}+4}}{8} \\
& \left.-\frac{\delta_{n_{i}, 4-n_{j}}}{8}\right\} \delta_{m_{i}, m_{j}} \\
& I_{4}=n_{i} \pi \int_{u=0}^{1} \int_{v=0}^{1}\left(\frac{d^{2} v}{d y^{2}}\right) \frac{1}{\tan \left(n_{i} \pi v\right)} \\
& \times \phi_{i}(u, v) \phi_{j}(u, v) d u d v \\
& =\frac{n_{i}}{\alpha_{y}^{2}}\left\{\frac{\delta_{n_{i}, 2-n_{j}}}{4}+\frac{\delta_{n_{i}, n_{j}-2}}{4}-\frac{\delta_{n_{i}, n_{j}+2}}{4}\right. \\
& \left.-\frac{\delta_{n_{i}, 4-n_{j}}}{8}-\frac{\delta_{n_{i}, n_{j}-4}}{8}+\frac{\delta_{n_{i}, n_{j}+4}}{8}\right\} \delta_{m_{i}, m_{j}} \\
& I_{5}=m_{i} \pi \int_{u=0}^{1} \int_{v=0}^{1} 2\left(\frac{d u}{d x}\right)^{2} \frac{1}{\tan \left(m_{i} \pi u\right)} \\
& \times \phi_{i}(u, v) \phi_{j}(u, v) \frac{\partial \ln (n)}{\partial u} d u d v \\
& +\int_{u=0}^{1} \int_{v=0}^{1} 2\left(\frac{d u}{d x}\right)^{2} \phi_{i}(u, v) \phi_{j}(u, v) \\
& \times \frac{\partial^{2} \ln (n)}{\partial u^{2}} d u d v \\
& =\frac{1}{\alpha_{x}^{2}} \int_{u=0}^{1} d u \int_{v=0}^{1} d v\{\ln (n) \\
& \times\{\{16[c(2 u)-c(4 u)] \\
& \left.-m_{j}^{2}[c(4 u)-4 c(2 u)+3] s_{i}(u) s_{j}(u) s_{i}(v) s_{j}(v)\right\} \\
& +m_{i} m_{j}[c(4 u)-4 c(2 u)+3] c_{i}(u) c_{j}(u) s_{i}(v) s_{j}(v) \\
& -4 m_{i}[s(4 u)-2 s(2 u)] c_{i}(u) s_{j}(u) s_{i}(v) s_{j}(v) \\
& \left.\left.-8 m_{j}[s(4 u)-2 s(2 u)] s_{i}(u) c_{j}(u) s_{i}(v) s_{j}(v)\right\}\right\}
\end{aligned}
$$$$
I_{6}=\int_{u=0}^{1} \int_{v=0}^{1} 2\left(\frac{d^{2} u}{d x^{2}}\right) \phi_{i}(u, v) \phi_{j}(u, v) \frac{\partial \ln (n)}{\partial u} d u d v
$$$$
=\frac{2}{\alpha_{x}^{2}} \int_{u=0}^{1} d u \int_{v=0}^{1} d v\{\ln (n)
$$$$
\times\left\{4[c(4 u)-c(2 u)] s_{i}(u) s_{j}(u) s_{i}(v) s_{j}(v)\right.
$$$$
+m_{i}[s(4 u)-2 s(2 u)] c_{i}(u) s_{j}(u) s_{i}(v) s_{j}(v)
$$$$
\left.\left.+m_{j}[s(4 u)-2 s(2 u)] s_{i}(u) c_{j}(u) s_{i}(v) s_{j}(v)\right\}\right\} \text {. }
$$

In (33) and (34), sine related functions $s(2 u)=\sin (2 u)$, $s_{i}(u)=\sin \left(m_{i} \pi u\right), s_{i}(v)=\sin \left(n_{i} \pi v\right)$, etc. For cosine related functions, they are abbreviated by the symbol $c$.

\section{REFERENCES}

[1] C. H. Henry and B. H. Verbeek, "Solution of the scalar wave equation for arbitrarily shaped dielectric waveguides by two-dimensional Fourier analysis," J. Lightwave Technol., vol. 7, pp. 308-313, 1989.

[2] D. Marcuse, "Solution of the vector wave equation for general dielectric waveguides by the Galerkin method," IEEE J. Quantum Electron., vol. 28, pp. 459-465, 1992.

[3] S. J. Hewlett and F. Ladouceur, "Fourier decomposition method applied to mapped infinite domains: Scalar analysis of dielectric waveguides down to modal cutoff," J. Lightwave Technol., vol. 13, pp. 375-383, 1995.

[4] A. Weisshaar, J. Li, R. L. Gallawa, and I. C. Goyal, "Vector and quasivector solutions for optical waveguide modes using efficient Galerkin's method with Hermite-Gauss basis functions," J. Lightwave Technol., vol. 13, pp. 1795-1800, 1995.

[5] K. S. Chiang, "Review of numerical and approximate methods for the modal analysis of general optical dielectric waveguides," Opt. Quantum Electron., vol. 26, pp. S113-S134, 1994.

[6] A. W. Snyder and J. D. Love, Optical Waveguide Theory. London, U.K.: Chapman and Hall, 1983.

[7] E. Anderson, Z. Bai, C. Bischof, J. Demmel, J. Dongarra, J. Du Croz, A. Greenbaum, S. Hammarling, A. McKenney, S. Ostrouchov, and D. Sorensen, LAPACK Users's Guide, 2nd ed. Philadelphia, PA: S.I.A.M., 1995.

[8] G. M. Berry, S. V. Burke, C. J. Smartt, T. M. Benson, and P. C. Kendall, "Exact and variational Fourier transform methods for analysis of multilayered planar waveguides," Inst. Elect. Eng. Proc. J., vol. 142, pp. 66-75, 1995

[9] W. P. Huang and C. L. Xu, "Simulation of three-dimensional optical waveguides by a full-vector beam propagation method," IEEE $J$. Quantum Electron., vol. 29, pp. 2639-2649, 1993.

[10] T. Wolf, C. L. Shieh, R. Engelmann, K. Alavi, and J. Mantz, "Lateral refractive index step in GaAs/AlGaAs multiple quantum well waveguides fabricated by impurity-induced disordering," Appl. Phys. Lett., vol. 55, pp. $1412-1414,1989$.

[11] B. L. Weiss, Ed. "Quantum well mixing for optoelectronics," Opt. Quantum Electron., vol. 23, pp. S799-S994, 1991.

Kai Ming Lo received the Ph.D. degree in theoretical physics from the University of Sydney, Australia, in 1995.

He was a Postdoctoral Fellow of the Department of Electrical and Electronic Engineering, University of Hong Kong. His main research interests are waveguide modeling, optical fiber modal fields, and ion-implantation-defined waveguide.

E. Herbert Li (M'84-SM'95) received the Ph.D. degree in electronic engineering from the University of Surrey, Surrey, U.K.

$\mathrm{He}$ is currently Leader of the Optoelectronics Group at the Department of Electrical and Electronic Engineering, University of Hong Kong, and a Visiting Scholar at Harvard University. His current research interests are concerned with optoelectronic device fabrication and modeling, in particular, interdiffusion modified quantum wells for advanced performance optoelectronic devices. Since 1990, he has published over 130 papers, two book chapters, and three books in these areas.

Dr. Li is a "Distinguished Lecturer" of IEEE-EDS since 1997 and has served on several IEEE Committees. He is also an Editor of the International Journal of Optoelectronics and a Guest Editor of the IEEE JouRnal OF SELECTED Topics In Quantum Electroncs. He received the J. Langham Thompson Premium Prize in 1992 from the Institution of Electrical Engineers (IEE-U.K.) and his biography is published in Who's Who in Science and Engineering (Marquis, USA). 